\title{
Daily Changes in Ultraviolet Light Levels Can Synchronize the Circadian Clock of Bumblebees (Bombus terrestris)
}

\author{
Lars Chittka, Ralph J. Stelzer, and Ralf Stanewsky \\ School of Biological and Chemical Sciences, Queen Mary University of London, London, UK
}

\begin{abstract}
Endogenous circadian clocks are synchronized to the 24-h day by external zeitgebers such as daily light and temperature cycles. Bumblebee foragers show diurnal rhythms under daily light:dark cycles and short-period freerunning circadian rhythms in constant light conditions in the laboratory. In contrast, during the continuous light conditions of the arctic summer, they show robust 24-h rhythms in their foraging patterns, meaning that some external zeitgeber must entrain their circadian clocks in the presence of constant light. Although the sun stays above the horizon for weeks during the arctic summer, the light quality, especially in the ultraviolet (UV) range, exhibits pronounced daily changes. Since the photoreceptors and photopigments that synchronize the circadian system of bees are not known, we tested if the circadian clocks of bumblebees (Bombus terrestris) can be entrained by daily cycles in UV light levels. Bumblebee colonies were set up in the laboratory and exposed to $12 \mathrm{~h}: 12 \mathrm{~h}$ UV + : UV- cycles in otherwise continuous lighting conditions by placing UV filters on their foraging arenas for $12 \mathrm{~h}$ each day. The activity patterns of individual bees were recorded using fully automatic radiofrequency identification (RFID). We found that colonies manipulated in such a way showed synchronized 24-h rhythms, whereas simultaneously tested control colonies with no variation in UV light levels showed free-running rhythms instead. The results of our study show that bumblebee circadian rhythms can indeed be synchronized by daily cycles in ambient light spectral composition. (Author correspondence: r.stanewsky@qmul.ac.uk)
\end{abstract}

Keywords: Constant light, Light entrainment, Radiofrequency identification (RFID)

\section{INTRODUCTION}

Endogenous molecular clocks are responsible for the behavioral and physiological rhythms that can be observed in many different organisms (Bell-Pedersen et al., 2005; Young \& Kay, 2001). In nature, these clocks are normally synchronized to the 24-h day via external cues, or zeitgebers, such as daily light:dark changes and corresponding temperature cycles, but they are also able to sustain self-contained rhythms in constant laboratory conditions (Aschoff, 1979; Glaser \& Stanewsky, 2005). However, under such "free-running" conditions, clocks reveal their endogenous circadian period length $(\tau)$, which is usually slightly shorter or longer than $24 \mathrm{~h}$.

The molecular mechanisms underlying these internal rhythms and their entrainment by external cues are best understood in the fruit fly Drosophila melanogaster (Helfrich-Förster, 2005; Rosato et al., 2006; Stanewsky, 2002). Photic entrainment in Drosophila is largely accomplished by the flavoprotein cryptochrome (dCRY), which acts as a circadian photoreceptor (Emery et al., 1998, 2000b; Stanewsky et al., 1998). As long as a fly is exposed to light,
dCRY inhibits the start of a new cycle of the molecular clock in a light-dependent manner, resulting in arrhythmic behavior of Drosophila in constant continuous light conditions (LL) (Emery et al., 2000a; Helfrich-Förster, 2002; Konopka et al., 1989; Stanewsky, 2002).

In contrast to Drosophila, honeybees (Apis mellifera) and bumblebees (Bombus terrestris) stay rhythmic in LL and show free-running circadian rhythms under such conditions (Frisch \& Aschoff, 1987; Moore \& Rankin, 1985; Spangler, 1972; Stelzer et al., 2010b). These findings suggest that cryptochrome is not responsible for the photic entrainment of their clocks. The decoding of the honeybee genome revealed that honeybees do have a cryptochrome gene, but in contrast to Drosophila it encodes a more vertebrate-like protein (CRY2), which again in contrast to dCRY-is not photosensitive (Rubin et al., 2006; Yuan et al., 2007). Thus, CRY2 might be required for a working clock in bees, most likely as a transcriptional repressor. The question of how light synchronizes the circadian system of bees remains unanswered (Yuan et al., 2007).

Submitted April 2, 2012, Returned for revision May 6, 2012, Accepted September 12, 2012

Address correspondence to Ralf Stanewsky, School of Biological and Chemical Sciences, Queen Mary University of London, Mile End Road, London E1 4NS, UK. Tel.: +44(0)2078827718; Email: r.stanewsky@qmul.ac.uk 
A recent field study in the Arctic found that bumblebees show robust diurnal 24-h rhythms in their foraging patterns during the arctic summer (Stelzer \& Chittka, 2010). Despite the fact that bright daylight is available for $24 \mathrm{~h}$ for several weeks during the arctic summer season, the workers displayed little foraging activity between 23:00 and 08:00, which indicates that some external zeitgeber must have entrained their molecular clocks. In addition to cues such as daily cycles in light intensity and the corresponding temperature changes, daily cycles in light quality, especially in the ultraviolet (UV) range, could also be decisive. Like other bees, bumblebees are UV-blue-green trichromats, meaning that they can see UV light (Chittka, 1996; Kevan et al., 2001; Peitsch et al., 1992; Skorupski et al., 2007). Bees use UV light for a variety of tasks. Many flowers, for example, have UV-reflecting parts, which bees use for flower detection and discrimination (Chittka \& Raine, 2006; Daumer, 1958; Spaethe et al., 2001). Furthermore, the pattern of polarized light in the UV range allows bees to orientate themselves and to navigate between the nest and flower patches, even when the sun is not visible during cloudy days (von Frisch, 1949; Wehner, 1984). Thus, UV reception is crucial for multiple behavioural domains in bees.

Levels of UV radiation drop drastically during arctic summer nights. Although the sun always remains above the horizon during the arctic summer, its altitude during the 'night' can be very low. The lower the solar altitude, the lower is the UV radiation that reaches the Earth, since the distance the sun light needs to travel through the atmosphere before it reaches the Earth's surface is longer and thus a higher level of UV radiation is absorbed and scattered by ozone, water vapor, and other atmospheric components (Gates, 1966). This results in significant daily changes of available UV levels, which are more pronounced than the corresponding daily changes in absolute light intensity (Krüll, 1976) and might therefore be able to trigger rhythmic wavelength-dependent behavior (Hut et al., 2000; Krüll, 1976; Nuboer et al., 1983; Pohl, 1999; Stelzer \& Chittka, 2010). A new photoreceptor organ that expresses UV-sensitive opsins has recently been discovered in bumblebees in the lamina, a brain area that has been associated with circadian clock activity in other insects (Spaethe \& Briscoe, 2005). It is therefore possible that the molecular clock of bumblebees can be entrained by daily cycles in UV radiation levels, which could explain the robust diurnal rhythms in their foraging patterns observed during the arctic summer (Stelzer \& Chittka, 2010). Spectral cues have recently been shown to control circadian activity patterns in fish (Pauers et al., 2012), and UVlight cycles have been shown to play an important role in the entrainment of the rodent circadian pacemaker (e.g., van Oosterhout et al., 2012).

To test the role of such cues for bumblebee circadian rhythms, the foraging patterns of the species Bombus terrestris were observed in the laboratory under continuous light conditions, with UV light being available only for
$12 \mathrm{~h}$ each day. During the other $12 \mathrm{~h}$, UV light was blocked from entering the foraging arenas by placing UV-absorbing filters on top of them. The results indicate that daily changes in light quality are indeed able to entrain the circadian clocks of bumblebees.

\section{MATERIALS AND METHODS}

The experimental protocols used in this study are conform with the international ethical standards (Portaluppi et al., 2010).

\section{Experimental Colonies}

We tested 10 Bombus terrestris colonies, 6 using radiofrequency identification (RFID) tagging and 4 using an infrared (IR) light barrier placed at the entry tunnel to the nest box. All 10 colonies were obtained from a commercial breeder (Syngenta Bioline Bees, Weert, The Netherlands) shortly before they were used. Each colony contained between 50 and 80 workers and a queen upon arrival. The colonies were transferred into bipartite plywood nest boxes $(28 \mathrm{~cm} \times 16 \mathrm{~cm} \times 11 \mathrm{~cm})$ shortly after arrival. The nest boxes had small meshcovered holes $(\emptyset=2 \mathrm{~cm})$ in their side walls to allow for ventilation. The boxes were connected to foraging arenas $(60 \mathrm{~cm} \times 40 \mathrm{~cm} \times 30 \mathrm{~cm})$ via transparent Plexiglas tunnels (Chittka \& Thomson, 1997). All experiments were performed in windowless rooms.

\section{Monitoring Circadian Rhythms With Radiofrequency Technology}

The RFID experiments were split into two blocks (A and B). In each block three colonies were tested simultaneously (A1 to A3 and B1 to B3, respectively). During the experiments, the bees were fed on $50 \%$ sucrose solution $(v / v)$ provided ad libitum through standard birdcage feeders/fountains (fountain and feeder 5415; TRIXI Heimtierbedarf, Tarp, Germany) mounted to the outside walls of the arenas (Stelzer et al., 2010b). Thus, the feeders could be refilled without disturbing the bees. The foragers were able to reach the feeders either by flying or by walking up the arena wall. Two of these feeders were attached to every arena. Pollen was provided directly into the nest box every other day at random times of day to avoid it becoming a temporal cue for the bees. Illumination was provided by high-frequency lighting (TMS 24F lamps with HF-B 236 TLD [4.3 kHz] ballasts; Philips, Amsterdam, The Netherlands). A single fitting for two tubes held one Activa daylight fluorescent tube (Osram, Munich, Germany) and one UV blacklight fluorescent tube (58 W; BLT Direct, Ipswich, UK). Light intensities in the four foraging arenas varied between 2168 and 2435 lux (measured at the bottom of the foraging arenas using a PCE-L335 Lux Meter; PCE Group, Meschede, Germany). The occasions when a researcher entered the experimental room, to feed the bees and download the data from the RFID readers (see below), were reduced to an absolute minimum (once or twice a day 


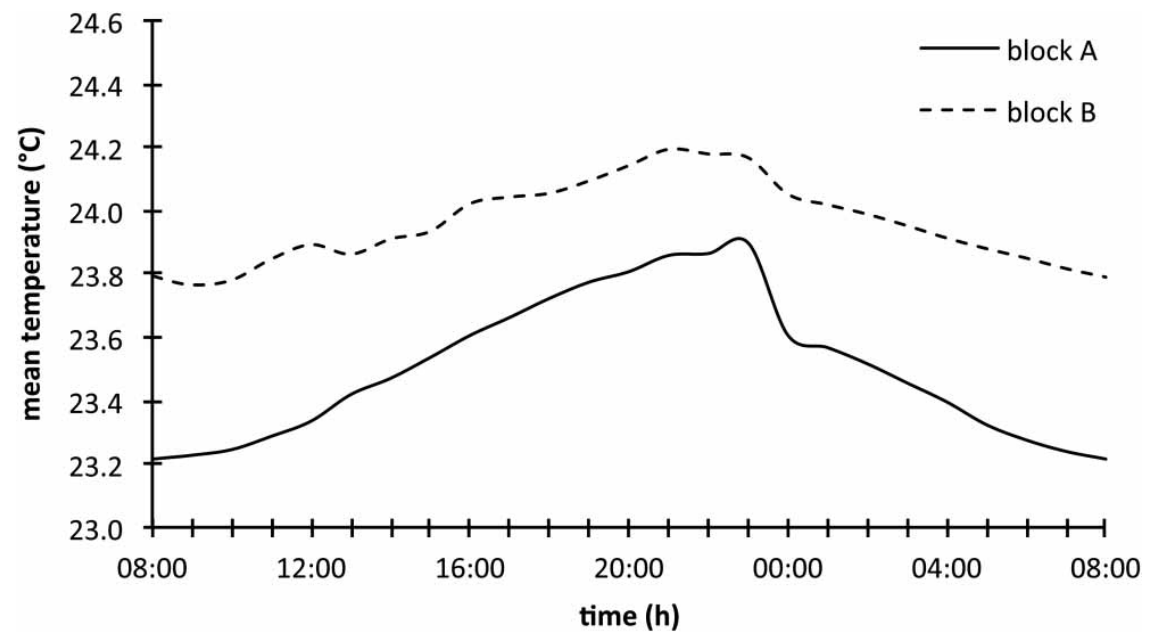

FIGURE 1. Mean hourly ambient temperature $\left({ }^{\circ} \mathrm{C}\right)$ in the experimental room during the RFID experiments in block A and block B. Temperature fluctuations varied between $.7^{\circ} \mathrm{C}$ and $2.0^{\circ} \mathrm{C}$ (mean: $1.1^{\circ} \mathrm{C} \pm .2^{\circ} \mathrm{C}$ ) in block $\mathrm{A}$ and between $.4^{\circ} \mathrm{C}$ and $1.5^{\circ} \mathrm{C}\left(\mathrm{mean}: .9^{\circ} \mathrm{C} \pm .3^{\circ}\right.$ C) in block B.

at random, except when the filters needed to be changed at certain times).

To monitor the complete foraging activity of individual bees, radiofrequency identification (RFID) was used (Molet et al., 2008; Stelzer et at., 2010a, 2010b; Streit et al., 2003). Small RFID tags $(1.0 \mathrm{~mm} \times 1.6 \mathrm{~mm} \times$ $0.5 \mathrm{~mm}$; mic3-TAG 64 bit RO, iID2000, $13.56 \mathrm{MHz}$ system; Microsensys, Erfurt, Germany) were glued to the dorsal surface of the thorax of the bees. An RFID reader (iID2000, 2k6 HEAD; Microsensys) was integrated into the tunnel close to the nest entrance. All bees were allowed to leave and enter the nest freely during the experiments. The RFID reader automatically recorded date and time when a tagged worker passed it, as well as the identity of the passing bee. The data were downloaded from the RFID readers every day at different times of the day, except the last $8 \mathrm{~d}$ of each block, when the data were downloaded each day when the light filters were changed (see below).

Room temperature was automatically recorded every other minute (block A) or every minute (block B) using a data logger (HOBO U10-00 Temperature Data Logger; Onset Computer, Pocasset, MA, USA). Temperature fluctuated daily between $0.7^{\circ} \mathrm{C}$ and $2.0^{\circ} \mathrm{C}$ (block A) or between $0.4^{\circ} \mathrm{C}$ and $1.5^{\circ} \mathrm{C}$ (block B) (Figure 1).

\section{Light Regimes}

Colonies were first kept under a $12 \mathrm{~h}: 12 \mathrm{~h}$ light:dark light regime for $7 \mathrm{~d}$, followed by $7 \mathrm{~d}$ of $\mathrm{LL}+\mathrm{UV}$ before the observations with rhythmic UV schedules began. These observations lasted $8 \mathrm{~d}$ and were carried out in continuous light conditions. UV-light cycles were generated by placing a UV filter (Arri 226; Munich, Germany) on the foraging arenas of colonies A2 and B2 for $12 \mathrm{~h}$ each day (colony A2: from 09:00 to 21:00; colony B2: from 10:00 to 22:00). To control for the possibility that raised levels of subtle mechanosensory cues resulting from researchers' activities in adjacent rooms during the day might be responsible for any observed rhythmicity in the foraging behavior of the bees, the foraging arenas of colonies A3 and B3 were shielded from UV during the opposite time periods (colony A3: from 21:00 to 09:00; colony B3: from 22:00 to 10:00). The illumination of the foraging arena of the third colony in each block (Al and B1, respectively) was not manipulated (i.e., constant) to control for any effects resulting from the observer entering the experimental room for applying the light filters and downloading the RFID data. To ensure that the four colonies exposed to UV cycles only received UV light while foraging in the arena, UV light was blocked from reaching the nest boxes and connection tubes by shielding them with UV filters around the clock.

\section{Data Analysis for RFID-Tagged Bees}

The level of colony activity was analyzed in 60-min bins. The resolution on the individual forager level was chosen as $1 \mathrm{~min}$, i.e. for each hour the number of minutes in which a given tagged bee passed the reader at least once was counted and used as level of activity within that hour. The raw data downloaded from the RFID readers were processed accordingly, using macros (Virtual Basic for Applications [VBA]) developed by the authors in Microsoft Excel. The processed data were then used for the circadian analysis on both the colony and the individual level in MATLAB (version R2007a; The MathWorks, Natick, MA, USA), using existing functions for Drosophila (Landskron et al., 2009; Levine et al., 2002a), which were adjusted to enable processing of the bumblebee data. The actual algorithms for the circadian analysis were not changed. Only bees that were active during at least six consecutive days during the UV observations were considered for the analysis. Actograms and daily average histograms were plotted in MATLAB. The free-running periods were calculated by autocorrelation analysis performed in MATLAB (Levine et al., 2002b). This function also determines the statistical 
significance of the period values (and thereby that of overall rhythmicity) indicated by the RI (rhythmicity index). The RI value reflects the height of the third peak of the autcorrelogram and if RI is greater than the $95 \%$ confidence interval, the period value detected by autocorrelation is significant. The RS (rhythmicity statistics) value used in the current study is obtained from the ratio of RI value to the $95 \%$ confidence line and bees with an RS value of $\geq 1$ were considered rhythmic and used for the circadian analysis on the colony level (Levine et al., 2002a, 2002b).

\section{Monitoring Colony-Level Foraging Activity With IR Light Barriers}

A second experiment with a further four colonies (C, D, E, and F) was performed to ensure the following: that (a) temperature could not be a factor in entrainment; (b) UV intensity rather than overall light intensity was the crucial factor in determining diel patterns; and (c) any rhythmicity observed in the experiments above was indeed an effect of entrainment of the circadian clock to UV light and not by a direct effect of the light changes (masking). In these experiments, overall colony foraging activity was measured by a battery-powered IR light barrier placed in the entrance/exit tunnel that linked the nest box with the foraging arena. This was connected to a Hobo U11-001 3 State/1 Event Data Logger (Measurement Systems, Newbury, UK). These bee counters were provided courtesy of Remco Huvermann of Koppert Biological Systems (Berkel en Rodenrijs, Netherlands). Experiments were performed in a temperature-controlled room and to ensure that temperature was indeed stable, we performed measurements every 5 min over a period of $5 \mathrm{~d}$, using a Tinytag TG-4080 temperature logger (Gemini Data Loggers, Chichester, UK). The average temperature was $21.75^{\circ} \mathrm{C}$ (minimum $21.62^{\circ} \mathrm{C}$; maximum $21.86^{\circ} \mathrm{C}$; so the maximal variation in the room was negligible at $0.24^{\circ} \mathrm{C}$ ). To minimize any form of mechanical stimulation, these colonies were provided with ad libitum food quantities that never needed topping up during the entire experiment. We placed a 2.5-L container of carbohydrate solution into each foraging arena (these containers come with the bee colonies as provided by Syngenta Bioline Bees). Pollen provisions were prepared by mixing frozen pollen with just enough honey to generate a highly viscous paste; $100 \mathrm{~mL}$ of this paste were provided directly into the colony before experiments began.

Colonies were placed in LL + UV conditions for $1 \mathrm{wk}$ before experiments began. Their activity was subsequently monitored for another $7 \mathrm{~d}$ under permanent LL + UV conditions. As in the RFID experiment above, illumination was provided by one Activa daylight fluorescent tube and one UV blacklight fluorescent tube. So that intensity could be controlled separately for the daylight tube, both lights were placed in separate fittings. During LL + UV conditions, a Rosco 3403 (Sun N6) neutral density filter was placed immediately beneath the daylight tube fitting. This filter transmits less than
$25 \%$ of all incident light over the entire bee visual spectrum; it was used to attenuate the intensity of the daylight tube, and thus to ensure that overall light intensity in UV+ conditions was not higher than in UV- conditions. On the eighth day ("transition day"), at 21:00, a UV filter (Arri 226) was placed on top of each flight arena, and the neutral density filter in front of the daylight tube was removed. From then on for $7 \mathrm{~d}, \mathrm{UV}+$ conditions were established at 9:00, and UV- conditions were established at 21:00. On day 8 (the second "transition day"), LL + UV conditions were reestablished at 9:00, and these conditions were then retained for another full week, to explore the possibility that any rhythmicity in the previous week might have been generated by masking. Data were collected in 60-min bins and actograms for each colony were plotted using the ActogramJ plug-in for ImageJ (National Institutes of Health, Bethesda, MD, USA) after normalization for variable colony size (Schmid et al., 2011). Period values ( $\tau$ ) and rhythmicity were also calculated in ActogramJ using $\chi^{2}$ analysis.

\section{RESULTS}

Observation and Analysis of Colony Behavior Using RFID

Out of approximately 500 workers that were tagged in total, the data of 245 bees met the criteria for being included in the analysis of the UV observations (see Table 1 and Materials and Methods for more detail). The proportions of rhythmic bees in each colony varied from $36.4 \%$ to $84.4 \%$ during the different conditions (Table 1). Plotting the average activity of all rhythmic

TABLE 1. Summary of rhythmic colony behavior during the different light regimes

\begin{tabular}{llccc}
\hline Colony & Light regime & $\mathrm{n}$ & \% rhythmic & $\tau \pm$ SEM (h) \\
\hline A1 & LD & 62 & 79.0 & $23.6 \pm .1$ \\
& LL & 91 & 69.2 & $22.9 \pm .2$ \\
\multirow{4}{*}{ A2 } & LL & 66 & 50.0 & $22.5 \pm .2$ \\
& LD & 32 & 84.4 & $23.7 \pm .1$ \\
& LL & 54 & 61.1 & $23.1 \pm .3$ \\
A3 & UV & 47 & 44.7 & $22.8 \pm .3$ \\
& LD & 42 & 57.1 & $24.0 \pm .2$ \\
& LL & 71 & 60.6 & $23.0 \pm .3$ \\
B1 & UV & 40 & 50.0 & $23.7 \pm .3$ \\
& LD & 26 & 73.1 & $23.3 \pm .2$ \\
& LL & 34 & 61.8 & $22.9 \pm .4$ \\
B2 & LL & 35 & 71.4 & $23.0 \pm .2$ \\
& LD & 34 & 73.5 & $23.8 \pm .2$ \\
& LL & 43 & 46.5 & $23.4 \pm .3$ \\
B3 & UV & 36 & 55.6 & $23.5 \pm .4$ \\
& LD & 14 & 50 & $23.5 \pm .3$ \\
& LL & 22 & 36.4 & $23.8 \pm .4$ \\
& UV & 21 & 61.9 & $24.0 \pm .4$ \\
\hline
\end{tabular}

For each colony, the table displays the different light conditions (12 h:12 h light:dark cycles [LD]; constant light [LL]; 12-h light + UV:12-h light - UV [UV]), the number (n) and proportion of rhythmic bees (\% rhythmic bees), and the mean period $(\tau)$ of all rhythmic bees (mean \pm SEM). 
Col A1 (LL)

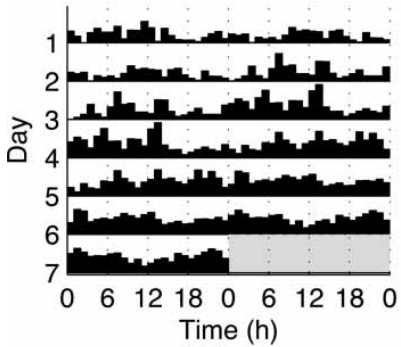

Col A2 (UV)

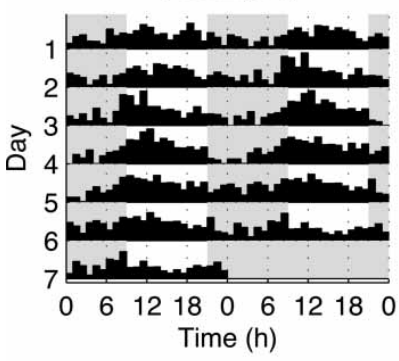

Col A3 (UV)

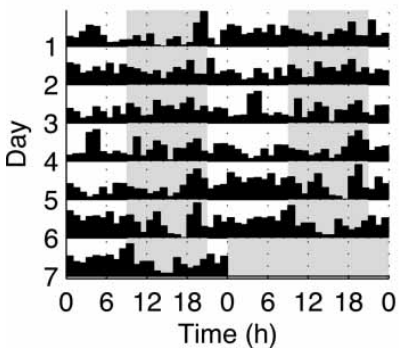

Col B1 (LL)

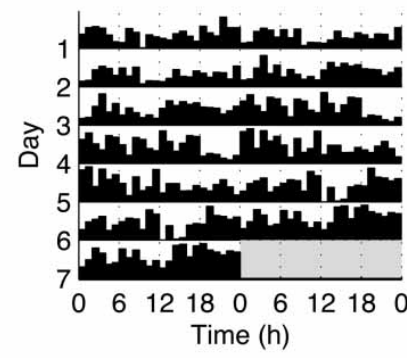

Col B2 (UV)

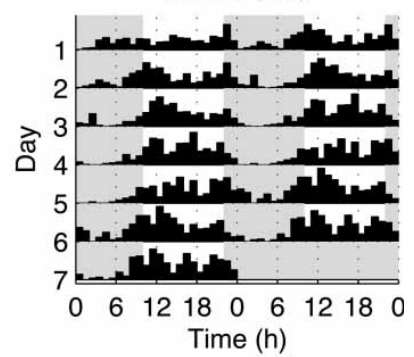

Col B3 (UV)

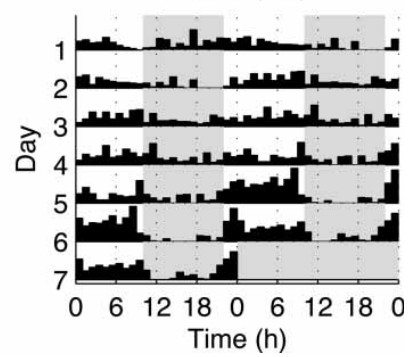

FIGURE 2. Double plotted actograms of the eight colonies tested in two blocks (A and B) during the UV observations in continuous light conditions. A1 and B1: nonmanipulated control colonies, which had continuous access to UV light. A2 and B2: colonies whose foraging arenas were covered with a UV filter between 21:00 and 09:00 and between 22:00 and 10:00, respectively, indicated by the shaded areas. A3 and B3: colonies whose foraging arenas were covered with a UV filter between 09:00 and 21:00 and between 10:00 and 22:00, respectively (shaded areas). Each row represents two consecutive days of the experiment; each bar corresponds to $1 \mathrm{~h}$ of the day and the height of the bars indicates the level of foraging activity in that hour.

individuals from each colony as actograms revealed that the nonmanipulated control colonies (Al and B1) showed no temporally organized activity peaks (Figure 2), consistent with different free-running periods and activity phases of individual bees under constant lighting conditions (LL). In contrast, all four colonies that were exposed to daily variations in UV light exhibited temporally organized activity with peaks occurring during UV exposure (Figure 2). In one of the colonies (A3), the daily rhythms were less strongly pronounced, although even in that colony, activity was higher during intervals with access to UV light (Figures 2, 3), and an individual-level analysis (see below) clearly shows the effects of daily cycles of UV illumination also in this colony. These results suggest that the daily 12 -h exposure to UV light indeed synchronizes activity of bumblebees.
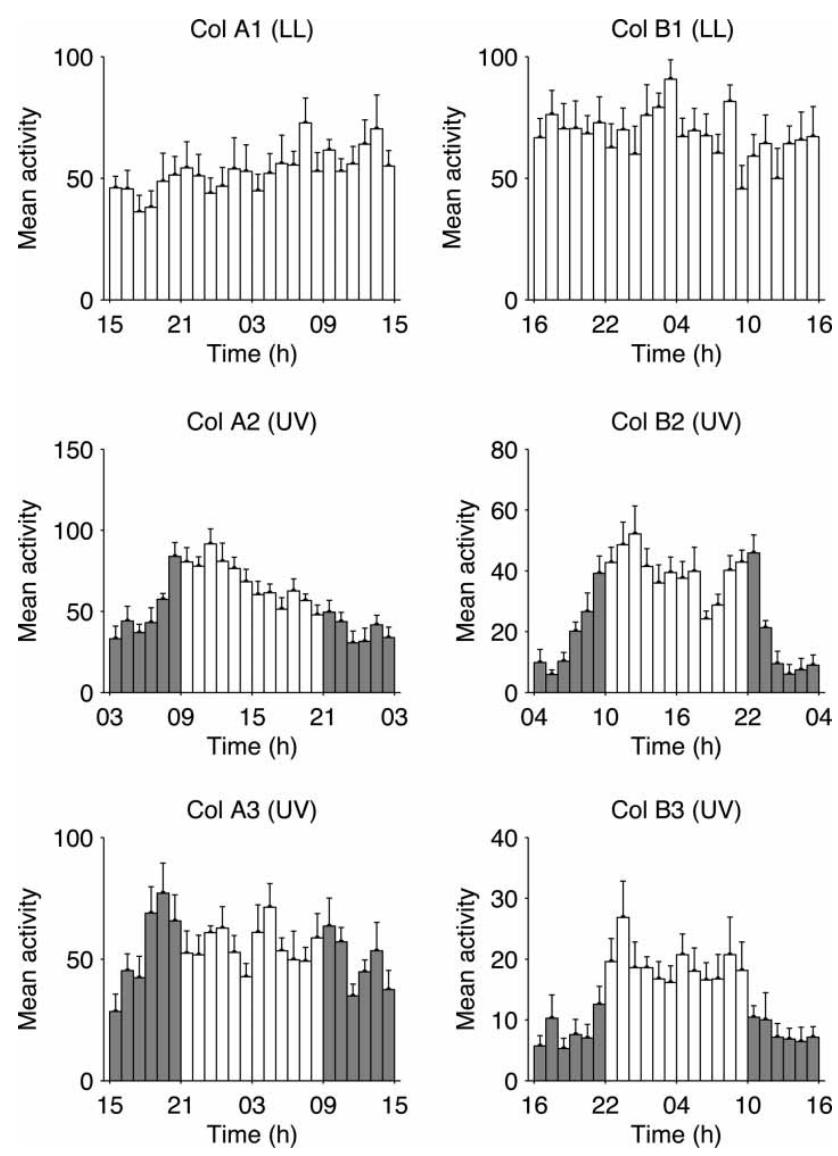

FIGURE 3. Daily average plots of the foraging activity of the eight tested colonies during the $7 \mathrm{~d}$ they were manipulated with filters. Gray bars indicate when the UV filter was applied to the foraging arenas. Conditions are identical to those described in the legend to Figure 1. Each bar represents an hour of the day and the height of the bars indicates the level of activity. Error bars indicate SEM.

To better visualize the colony behavior during the exposure to UV cycles, we also calculated and plotted the average activity for this part of the experiment only (Figure 3; each histogram shows the average daily colony activity during the $7 \mathrm{~d}$ the bees were exposed to UV light intensity cycles). As expected, behavioral activity of the control colonies $\mathrm{Al}$ and $\mathrm{Bl}$, which were constantly exposed to UV light, did not show any discernible activity peaks (Figure 3). In contrast, all colonies exposed to daily variation in UV light showed higher activity levels during the time they were exposed to UV light. Moreover, they started to increase their activity levels $1-2 \mathrm{~h}$ before UV exposure (Figure 3). In Drosophila and other organisms, this type of behavior is known as "anticipation" and serves as a clear indication for an underlying synchronization of the circadian clock (e.g. Hamblen-Coyle et al., 1992).

\section{Clock-Controlled Foraging Activity of Bumblebees Is Synchronized by UV-Light Cycles}

The results obtained with RFID-tagged bees showed that foraging activity was synchronized to the daily UV cycles and the anticipatory activity onsets before UV exposure 
suggested that the circadian clock driving this behavior was also synchronized by UV light. To confirm clock synchronization by UV-light cycles, we performed a second set of experiments, in which four bumblebee colonies were analyzed in constant conditions after being exposed to UV cycles. In this experiment, bees were not individually tagged and foraging activity of worker bees was measured by placing an IR light detector between the nest and foraging arena (see Material and Methods). Bumblebees were first exposed to $7 \mathrm{~d}$ of $\mathrm{LL}$ in the continuous presence of UV light. Three of four colonies (C, E, and F) were arrhythmic (Figure 4) during the initial LL phase as one might expect. Surprisingly, one colony (D) showed significant rhythmic activity $(\tau=22$

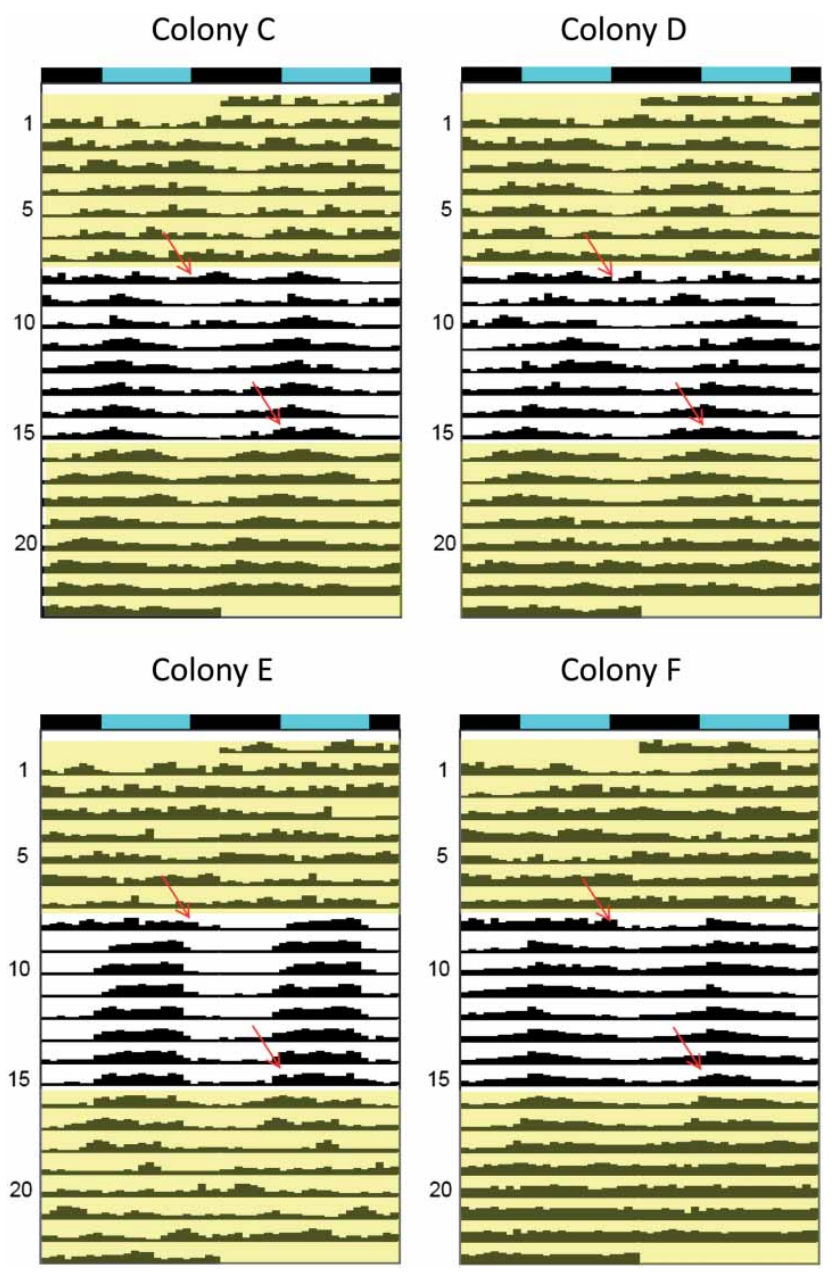

FIGURE 4. Colony behavior in constant light in the absence or presence of UV cycles. Double plotted average actograms of 4 colonies tested in LL followed by LL + UV cycles and final release into constant conditions (LL). Yellow or grey areas: LL without UV cycles; no shading (days 8 to 15): LL + UV cycles as indicated by the environmental bar above each actogram (blue or grey: UV light on; black: UV light off). Arrows indicate when the UV filters were initially applied and removed for good, respectively. Note that all four colonies display synchronized activity rhythms during UV cycles and at least for 2-3 d in the subsequent constant conditions. Colonies were tested in two sequential blocks ( $\mathrm{C}$ with $\mathrm{D}$ and $\mathrm{E}$ with $\mathrm{F}$ ) in a temperature-controlled room. Period values and rhythmicity during constant and UV-cycle conditions were determined using $\chi^{2}$ periodogram analysis $(p<0.05)$. h) during $3 \mathrm{~d}$ in the middle of the initial LL exposure (periods and rhythmicity were determined by $\chi^{2}$ periodogram analysis, $p<.05$ ), presumably as a result of an aftereffect of LD exposure before the colony arrived in the laboratory. However, even this colony behaved arrhythmic on the $2 \mathrm{~d}$ before entering the UV cycles, showing that by the beginning of UV cycles, none of the colonies retained any rhythmicity. Similar to the results shown for RFID experiments (Figure 2), all four colonies synchronized their activity to the UV cycles ( $\tau=24 \mathrm{~h}$ for colonies $\mathrm{C}, \mathrm{E}$, and $\mathrm{F}$ and $23 \mathrm{~h}$ for colony $\mathrm{D}$ ), being mainly active during the $12 \mathrm{~h}$ of UV light exposure. In the final part of the experiment, colonies were again exposed to LL in the constant presence of UV light. If the circadian clock in individual bees was synchronized by the rhythmic exposure to UV light, we should be able to observe synchronized activity at the colony level at least for a limited amount of time (before the different individual free-running periods obscure these rhythms). Alternatively, if the observed rhythmicity during the UVcycle exposure is directly driven by these cycles-bypassing the circadian clock (masking), we should expect arrhythmic behavior at the colony level immediately after transfer to constant conditions, because the circadian clocks of individual bees would not be synchronized. All four colonies exhibited clear rhythmicity upon transfer to constant conditions and varied only in the time they stayed synchronized (colony $\mathrm{C}$ for $7 \mathrm{~d}, \tau=$ $23 \mathrm{~h}$; colony D for $4-5 \mathrm{~d}, \tau=24 \mathrm{~h}$; colonies $\mathrm{E}$ and $\mathrm{F}$ for 3 d, $\tau=23$ and $24 \mathrm{~h}$, respectively; Figure 4 ). We therefore conclude that the circadian clock in individual forager bees must have been synchronized during (and by) the exposure to UV cycles.

\section{Analysis of Colony Subgroups and Individual Bees (RFID- Monitored Colonies)}

Although the colony-level analysis showed that UV light is able to synchronize bumblebee behavioral rhythms, one of the colonies (A3) showed less pronounced rhythmic behavior. A possible explanation for this variation between colonies could be that not all bees within each colony are synchronized by daily fluctuations in UV light alone; in other words, cycles of UV light could represent a weaker zeitgeber compared with full-spectrum LD cycles. Since colony-level analyses "average out" variation of individual rhythmicity, we also evaluated the number of individuals that synchronized to UV-light variation by comparing their period length under freerunning and UV-cycling conditions using our RFIDtagged bees. If the endogenous free-running period $(\tau)$ of an individual is shorter or longer than $24 \mathrm{~h}$, upon transfer to UV cycles it will either keep this period if it does not synchronize to the UV cycle, or adjust its period to that of the UV cycle $(\tau=24 \mathrm{~h})$ in case it does synchronize. Since most bees show a short period under free-running conditions (Stelzer et al., 2010b), we should be able to judge if synchronization occurs by determining the period values during the UV cycle. For 
TABLE 2. Distribution and alterations of period length $(\tau)$ values during the different experimental conditions

\begin{tabular}{|c|c|c|c|c|c|c|c|}
\hline Colony & $\tau$ & $\mathrm{LL}(\mathrm{n}=63)$ & LL2 (n = 33) & Colony & $\tau$ & $\operatorname{LL}(\mathrm{n}=21)$ & LL2 $(n=25)$ \\
\hline \multirow[t]{4}{*}{ Al } & $\tau<23.5$ & $61.9 \%$ & $78.8 \%$ & \multirow[t]{4}{*}{ B1 } & $\tau<23.5$ & $52.4 \%$ & $72.0 \%$ \\
\hline & $23.5 \leq \tau \leq 24.5$ & $30.2 \%$ & $15.2 \%$ & & $23.5 \leq \tau \leq 24.5$ & $33.3 \%$ & $20.0 \%$ \\
\hline & $\tau>24.5$ & $7.9 \%$ & $6.1 \%$ & & $\tau>24.5$ & $14.3 \%$ & $8.0 \%$ \\
\hline & $\tau$ & $\mathrm{LL}(\mathrm{n}=33)$ & $\pm \mathrm{UV}(\mathrm{n}=21)$ & & $\tau$ & $\operatorname{LL}(\mathrm{n}=20)$ & $\pm U V(n=20)$ \\
\hline \multirow[t]{4}{*}{ A2 } & $\tau<23.5$ & $66.7 \%$ & $52.4 \%$ & \multirow[t]{4}{*}{ B2 } & $\tau<23.5$ & $50.0 \%$ & $35.0 \%$ \\
\hline & $23.5 \leq \tau \leq 24.5$ & $21.2 \%$ & $42.9 \%$ & & $23.5 \leq \tau \leq 24.5$ & $35.0 \%$ & $55.0 \%$ \\
\hline & $\tau>24.5$ & $12.1 \%$ & $4.8 \%$ & & $\tau>24.5$ & $15.0 \%$ & $10.0 \%$ \\
\hline & $\tau$ & LL $(n=43)$ & $\pm U V(n=20)$ & & $\tau$ & $\operatorname{LL}(n=43)$ & $\pm \mathrm{UV}(\mathrm{n}=20)$ \\
\hline \multirow[t]{3}{*}{ A3 } & $\tau<23.5$ & $62.8 \%$ & $45.0 \%$ & \multirow[t]{3}{*}{ B3 } & $\tau<23.5$ & $37.5 \%$ & $23.1 \%$ \\
\hline & $23.5 \leq \tau \leq 24.5$ & $14.0 \%$ & $30.0 \%$ & & $23.5 \leq \tau \leq 24.5$ & $37.5 \%$ & $61.5 \%$ \\
\hline & $\tau>24.5$ & $23.3 \%$ & $25.0 \%$ & & $\tau>24.5$ & $25.0 \%$ & $15.4 \%$ \\
\hline
\end{tabular}

In each colony, bees were classified as having short $(<23.5 \mathrm{~h})$, normal $(24 \pm .5 \mathrm{~h})$, or long $(>24.5 \mathrm{~h})$ rhythms. Note that the percentage of bees with normal period increases during the UV cycles ( \pm UV) in all four colonies (A2, A3, B2, B3) exposed to these cycles compared with the preceding free-running (LL) conditions.

this, we divided each colony into three groups based on their period length during free-running conditions: (i) $\tau<23.5 \mathrm{~h}$; (ii) $23.5 \mathrm{~h}<\tau<24.5 \mathrm{~h}$; (iii) $\tau>24.5 \mathrm{~h}$ (Table 2). Strikingly, in all colonies exposed to UV cycles (A2, B2, A3, B3), the percentage of individual bees with periods close to $24 \mathrm{~h}$ (ii) doubled (A colonies) or almost doubled (B colonies) during exposure to UV cycles compared with free-running conditions, whereas the percentage of short-period bees dropped (Table 2). This was not the case for colonies Al and B1, which after initial LD entrainment were kept in constant conditions throughout the experiment, and in fact showed the opposite trend (Table 2). From this analysis we conclude that at least a subgroup of bees within each of the UV-cycle-exposed colonies synchronized their behavior to UV light.

Based on these data we should be able to identify individual bees that during the course of the experiment show a visible change of their behavioral period upon transfer from free-running to UV-cycling conditions. This was indeed the case, and Figure 5 shows examples of individuals exhibiting this alteration of period length (bees that synchronize; Figure 5B-D) compared with control bees that keep their free-running period (Figure 5A). Inspection of all individual actograms revealed that only 5 of the 125 bees of the Al and B1 colonies showed a spontaneous dramatic change of their free-running period during the constant light condition, indicating that most of the bees showing period alterations upon transfer to UV cycles indeed synchronized to these cycles.

\section{DISCUSSION}

The results of this study show that daily cycles in UV radiation are able to entrain the circadian clocks of bumblebees. Even though there was some variation in rhythmicity between the eight colonies exposed to UV cycles, in all colonies most of the activity occurred when UV light was present, and the analysis of subgroups and individuals of a subset of these colonies clearly indicate entrainment by UV cycles (Table 2, Figure 5).
Moreover, since the two nonmanipulated control colonies (Al and $\mathrm{B} 1$ ) showed no signs of synchronization at the colony or individual level, it is clear that neither any disturbances from adjacent rooms during the day nor entering the experimental room at certain times was responsible for the activity patterns found in the four UV-cycle-exposed colonies. We also ruled out that direct effects of UV light (masking) were responsible for the observed rhythmic behavior during UV cycles, because synchronized behavior at the colony level continued for several days in constant conditions after exposure to UV-light cycles (Figure 4). In a previous study, we had analyzed the interrelationship between colony and individual rhythms (Stelzer et al., 2010b). It was shown that after the transition from LD to LL, individuals show free-running rhythms with varying periods, which means that at the colony level, rhythms persist for a few days, and then deteriorate because individual variation in rhythmic activities becomes too pronounced to be detectable at the colony level. This means that colony-level activity can be used as a proxy to show entrainment during $\mathrm{LD}$ by evaluating the pattern of subsequent disintegration of colony-level rhythmicity under LL conditions.

Based on the entrainment achieved in colonies covered with UV filters at opposite times (A versus B colonies), the nonentrainment of the controls ( $\mathrm{Al}$ and $\mathrm{B} 1$ colonies), and the tight temperature control in the IR experiment (colonies $\mathrm{C}, \mathrm{D}, \mathrm{E}$, and F), it is also ruled out that small room temperature fluctuations during the RFID experiments (Figure 1) resulted in colony entrainment. Moreover, a close relative of the bumblebee, the honeybee, requires temperature cycles with amplitudes of $\sim 10^{\circ} \mathrm{C}$ (Moore \& Rankin, 1993) for behavioral synchronization, which is clearly above the temperature variations in the current study (Figure 1). It is likely, however, that other factors in addition to UV-light cycles influence the behavioral diurnal rhythms in nature and that they synergistically contribute to entrainment (Moore \& Rankin, 1993).

The question whether the synchronized behavior observed in the four UV-cycle-exposed colonies' activity 
A
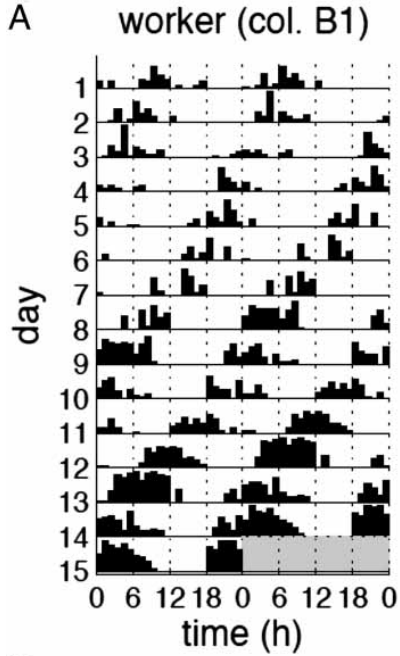

C

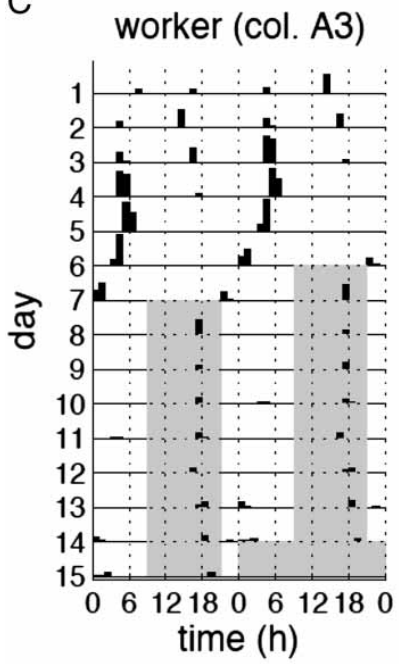

B worker (col. A2)

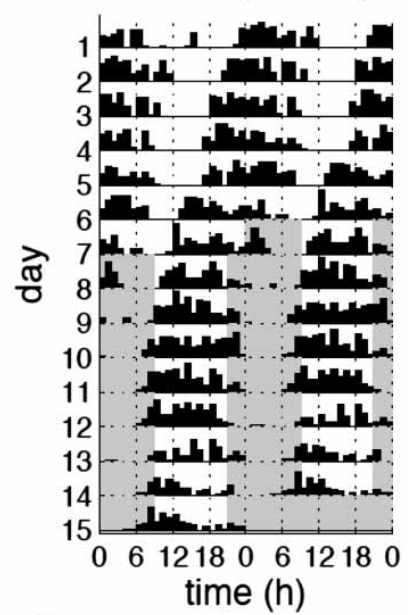

D worker (col. B2)

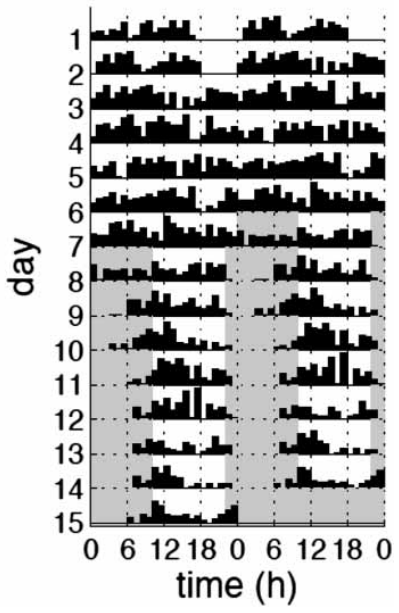

FIGURE 5. Exemplary double-plotted actograms of individual workers during the UV cycles and the preceding $7 \mathrm{~d}$ of LL, during which UV light was available for $24 \mathrm{~h}$. (A) Worker of one of the nonmanipulated colonies (B1) with a short free-running rhythm throughout the observation period. (B) Worker of colony A2, in which UV light was blocked between 21:00 and 09:00 during the UV observations (days 8 to 15), indicated by the shaded areas. This worker showed a short free-running rhythm during the first 7 $\mathrm{d}$ in $\mathrm{LL}$, which changed to a 24 -h period during the $\mathrm{UV}+: \mathrm{UV}-$ cycle. (C) Worker of colony A3, in which UV light was blocked between 09:00 and 21:00 during the UV observations. After a few days in LL, this bee showed a short free-running rhythm, which also lengthened to $24 \mathrm{~h}$ when the UV filter was applied daily. This particular bee was active during the time UV light was blocked (shaded area). (D) Worker of colony B2, which showed unusual arrhythmic behavior in LL, but switched to rhythmic behavior with a 24-h period during UV cycles.

patterns were exclusively a result of activating the bees' UV receptors, either extraocular ones or those in the compound eyes, cannot be answered with certainty. The UV filter applied here thoroughly abrogates light with wavelengths below $380 \mathrm{~nm}$ (Dyer \& Chittka, 2004), leaving no doubt that the bees have been exposed to UV cycles. However, the green- and blue-sensitive opsins, despite having their absorption maxima at longer wavelength, nevertheless also absorb in the UV

range (Kevan et al., 2001). In other words, by applying the UV filter we reduced light input to all three photoreceptors and not just the UV receptors. Nevertheless, we can conclude that the alternating absence and presence of UV light was sufficient to synchronize bumblebee behavior.

In conclusion, our results provide strong evidence that UV light is able to synchronize the circadian clock of bumblebees, consistent with the idea that natural UV cycles contribute to synchronization of bumblebee clocks north of the arctic circle (Stelzer \& Chittka, 2010).

\section{ACKNOWLEDGMENTS}

The authors would like to thank Oscar Ramos-Rodríguez for technical support, Taishi Yoshii for help with ActogramJ, and Syngenta Bioline Bees for providing the bumblebee colonies. We would also like to thank Remco Huvermann and Koppert Biological Systems for lending us bee counters and data loggers, and Tom Ings for a Tinytag temperature logger.

Declaration of Interest: This work has been supported by a Westfield Trust Studentship granted to R.J.S.

The authors report no conflicts of interest. The authors alone are responsible for the content and writing of the paper.

\section{REFERENCES}

Aschoff J. (1979). Circadian rhythms: influences of internal and external factors on the period measured in constand conditions. $Z$. Tierpsychol. 49:225-249.

Bell-Pedersen D, Cassone VM, Earnest DJ, Golden SS, Hardin PE, Thomas TL, Zoran MJ. (2005). Circadian rhythms from multiple oscillators: lessons from diverse organisms. Nat. Rev. Genet. 6:544-556.

Chittka L (1996). Does bee color vision predate the evolution of flower color? Naturwissenschaften 83:136-138.

Chittka L, Raine N E (2006). Recognition of flowers by pollinators. Curr. Opin. Plant Biol. 9:428-435.

Chittka L, Thomson J D (1997). Sensori-motor learning and its relevance for task specialization in bumble bees. Behav. Ecol. Sociobiol. 41:385-398.

Daumer K (1958). Blumenfarben, wie sie die Bienen sehen. Z. Vergl. Physiol. 41:49-110.

Dyer A G, Chittka L (2004). Bumblebee search time without ultraviolet light. J. Exp. Biol. 207:1683-1688.

Emery P, So W, Kaneko M, Hall J, Rosbash M (1998). CRY, a Drosophila clock and light-regulated cryptochrome, is a major contributor to circadian rhythm resetting and photosensitivity. Cell 95:669-679.

Emery P, Stanewsky R, Hall J, Rosbash M (2000a). A unique circadian rhythm photoreceptor. Nature 404:456-457.

Emery P, Stanewsky R, Helfrich-Forster C, Emery-Le M, Hall J, Rosbash M (2000b). Drosophila CRY is a deep brain circadian photoreceptor. Neuron 26:493-504.

Frisch B, Aschoff J (1987). Circadian-rhythms in honeybees-entrainment by feeding cycles. Physiol. Entomol. 12:41-49.

Gates D M (1966). Spectral distribution of solar radiation at the Earth's surface. Science 151:523-529.

Glaser F, Stanewsky R (2005). Temperature synchronization of the Drosophila circadian clock. Curr. Biol. 15:1352-1363. 
Hamblen-Coyle M J, Wheeler D A, Rutila J E, Rosbash M, Hall J C (1992). Behavior of period-altered circadian rhythm mutants of Drosophila in light:dark cycles. J. Insect Behav. 5:417-446.

Helfrich-Förster C (2002). The circadian system of Drosophila melanogaster and its light input pathways. Zoology 105:297-312.

Helfrich-Förster C (2005). Neurobiology of the fruit fly's circadian clock. Genes Brain Behav. 4:65-76.

Hut R A, Scheper A, Daan S (2000). Can the circadian system of a diurnal and a nocturnal rodent entrain to ultraviolet light? $J$. Comp. Physiol. A 186:707-715.

Kevan P G, Chittka L, Dyer A G (2001). Limits to the salience of ultraviolet: lessons from colour vision in bees and birds. J. Exp. Biol. 204:2571-2580.

Konopka R, Pittendrigh C, Orr D (1989). Reciprocal behaviour associated with altered homeostasis and photosensitivity of Drosophila clock mutants. J. Neurogenet. 6:1-10.

Krüll F (1976). Zeitgebers for animals in the continuous daylight of high arctic summer. Oecologia 24:149-157.

Landskron J, Chen K, Wolf E, Stanewsky R (2009). A role for the PERIOD:PERIOD homodimer in the Drosphila circadian clock. PLoS Biol. 7:e3.

Levine J, Funes P, Dowse H, Hall J (2002a). Signal analysis of behavioral and molecular cycles. BMC Neurosci. 3:1.

Levine J, Funes P, Dowse H, Hall J (2002b). Advanced analysis of a cryptochrome mutation's effects on the robustness and phase of molecular cycles in isolated peripheral tissues of Drosophila. BMC Neurosci. 3:5.

Molet M, Chittka L, Stelzer R J, Streit S, Raine N (2008). Colony nutritional status modulates worker responses to foraging recruitment pheromone in the bumblebee Bombus terrestris. Behav. Ecol. Sociobiol. 62:1919-1926.

Moore D, Rankin M A (1985). Circadian locomotor rhythms in individual honeybees. Physiol. Entomol. 10:191-197.

Moore D, Rankin M A (1993). Light and temperature entrainment of a locomotor rhythm in honeybees. Physiol. Entomol. 18:271-278.

Nuboer J F W, Nuys W M, Steenbergen J C (1983). Colour changes in a light regimen as synchronizers of circadian activity. J. Comp. Physiol. A 151:359-366.

Pauers M J, Kuchenbecker J A, Neitz M, Neitz J (2012). Changes in the colour of light cue circadian activity. Anim. Behav. 83:1143-1151.

Peitsch D, Fietz A, Hertel H, de Souza J, Ventura D F, Menzel R (1992). The spectral input system of hymenopteran insects and their receptor-based colour vision. J. Comp. Physiol. A 170:23-40.

Pohl H (1999). Spectral composition of light as a Zeitgeber for birds living in the high arctic summer. Physiol. Behav. 67:327-337.

Portaluppi F, Smolensky M H, Touitou Y (2010). Ethics and methods for biological rhythm research on animals and human beings. Chronobiol. Int. 27:1911-1929.

Rosato E, Tauber E, Kyriacou C (2006). Molecular genetics of the fruitfly circadian clock. Eur. J. Hum. Genet. 14:729-738.

Rubin E B, Shemesh Y, Cohen M, Elgavish S, Robertson H M, Bloch G (2006). Molecular and phylogenetic analyses reveal mammalian- like clockwork in the honey bee (Apis mellifera) and shed new light on the molecular evolution of the circadian clock. Genome Res. 16:1352-1365.

Schmid B, Helfrich-Forster C, Yoshii T (2011). A new ImageJ plug-in "ActogramJ" for chronobiological analyses. J. Biol. Rhythms 26:464-467.

Skorupski P, Doring T F, Chittka L (2007). Photoreceptor spectral sensitivity in island and mainland populations of the bumblebee, Bombus terrestris. J. Comp. Physiol. A Neuroethol. Sens. Neural Behav. Physiol. 193:485-494.

Spaethe J, Briscoe A D (2005). Molecular characterization and expression of the UV opsin in bumblebees: three ommatidial subtypes in the retina and a new photoreceptor organ in the lamina. $J$. Exp. Biol. 208:2347-2361.

Spaethe J, Tautz J, Chittka L (2001). Visual constraints in foraging bumblebees: flower size and color affect search time and flight behavior. Proc. Natl. Acad. Sci. U. S. A. 98:3898-3903.

Spangler H (1972). Daily activity rhythms of individual worker and drone honey bees Hymenoptera-Apidae. Ann. Entomol. Soc. Am. 65:1073-1076.

Stanewsky R (2002). Clock mechanisms in Drosophila. Cell Tissue. Res. 309:11-26.

Stanewsky R, Kaneko M, Emery P, Beretta B, Wagner-Smith K, Kay S, Rosbash M, Hall J (1998). The cryb mutation identifies cryptochrome as a circadian photoreceptor in Drosophila. Cell 95:681-692.

Stelzer R J, Chittka L (2010). Bumblebee foraging rhythms under the midnight sun measured with radiofrequency identification. $B M C$ Biol. 8:93.

Stelzer R J, Chittka L, Ings T C (2010a). Winter active bumblebees (Bombus terrestris) achieve high foraging rates in urban Britain. PLOS ONE 5:e9559.

Stelzer R J, Stanewsky R, Chittka L (2010b). Circadian foraging rhythms of bumblebees monitored by radio-frequency identification. J. Biol. Rhythms 25:257-267.

Streit S, Bock F, Pirk C W, Tautz J (2003). Automatic life-long monitoring of individual insect behaviour now possible. Zoology 106:169-171.

van Oosterhout F, Fisher S P, van Diepen H C, Watson T S, Houben T, Vanderleest H T, Thompson S, Peirson S N, Foster R G, Meijer J H (2012). Ultraviolet light provides a major input to non-imageforming light detection in mice. Curr. Biol. 22:1397-1402.

von Frisch K (1949). Die Polarisation des Himmelslichtes als orientierender Faktor bei den Tänzen der Bienen. Experientia 4:142-148.

Wehner R (1984). Astronavigation in insects. Annu. Rev. Entomol. 29:277-298.

Young M W, Kay S A (2001). Time zones: a comparative genetics of circadian clocks. Nat. Rev. Genet. 2:702-715.

Yuan Q, Metterville D, Briscoe A, Reppert S (2007). Insect cryptochromes: gene duplication and loss define diverse ways to construct insect circadian clocks. Mol. Biol. Evol. 24:948-955. 